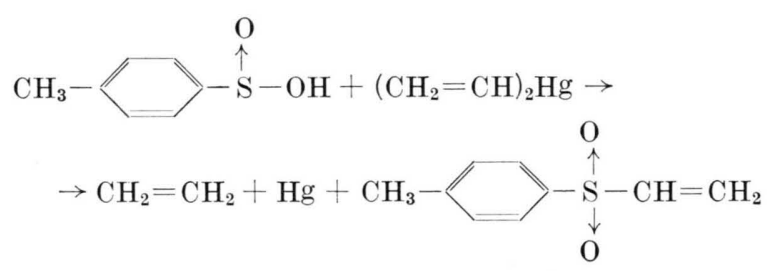

$\mathrm{C}_{9} \mathrm{H}_{10} \mathrm{O}_{2} \mathrm{~S}$ requires $\mathrm{C}, 59.32 ; \mathrm{H}, 5.53 ; \mathrm{S}, 17.60$.) The infrared spectrum showed the characteristic absorptions of a sulfone at 7.52 and $8.69 \mu^{9}$.

${ }^{9}$ L. J. Bellamy, "The Infrared Spectra of Complex Molecules", 2nd. Ed., Methuen, London 1956, p. 360.

\section{Organomercury Chemistry}

\section{Some Reactions of Vinyl- and Cyclopropyl- mercury Compounds}

\author{
By Erich Tobler and Donald J. Foster
}

Research Department, Union Carbide Olefins Company, Division of Union Carbide Corporation, South Charleston, West Virginia, U.S.A.

(Z. Naturforschg. 17 b, 136-137 [1962] ; eingeg. am 2. September 1961)

Vinyl- und Cyclopropylquecksilber-ester reagieren mit „Xanthogenaten“ unter Bildung der entsprechenden Alkylquecksilber-xanthogenate. Auf ähnliche Weise konnte aus Vinylquecksilber-acetat und Natriumthiocyanat Vinylquecksilber-thiocyanat dargestellt werden. Mit Acetylen setzen sich alkalische Lösungen von organischen Quecksilbersalzen zu Dialkylquecksilberacetylenen um.

Spengler and $W_{\text {eber }}{ }^{1}$, by treating several mercury addition products with potassium xanthate, obtained organomercury xanthates, which decomposed after a few days accompanied by the deposition of mercury sulfide. Under the same conditions, vinylmercuric and cyclopropylmercuric acetate react with potassium

$$
\begin{aligned}
& \mathrm{RHgO}_{2} \mathrm{CCH}_{3}+\mathrm{C}_{2} \mathrm{H}_{5} \mathrm{OC} \overbrace{\mathrm{SK}}^{\mathrm{S}} \\
& \stackrel{\mathrm{EtOH}}{\longrightarrow} \mathrm{C}_{2} \mathrm{H}_{5} \mathrm{OC} \underset{\mathrm{SHgR}}{\mathrm{S}}+\mathrm{CH}_{3} \mathrm{CO}_{2} \mathrm{~K}
\end{aligned}
$$

xanthate in ethanol to give vinylmercuric xanthate $\left(\mathrm{R}=\mathrm{CH}_{2}: \mathrm{CH}-\right)$, green-yellow plates, m. p. $40.5^{\circ}$ (Found: C, 17.37; H, 2.37; S, 18.58. $\mathrm{C}_{5} \mathrm{H}_{8} \mathrm{HgOS}_{2}$ requires $C, 17.21 ; \mathrm{H}, 2.29 ; \mathrm{S}, 18.35)$ and cyclopropylmercuric xanthate $(\mathrm{R}=\mathrm{Cyclopropyl})$, m. p. 87 to $88^{\circ}$, (Found: C, 19.92; H, 2.86; S, 17.41 . $\mathrm{C}_{6} \mathrm{H}_{10} \mathrm{HgOS}_{2}$ requires $\left.\mathrm{C}, 19.85 ; \mathrm{H}, 2.78 ; \mathrm{S}, 17.65\right)$, respectively. Both compounds show strong infrared absorption bands in the 8.0, 8.2, 9.0 and $9.7 \mu$ region. While cyclopropylmercuric xanthate exhibits considerable stability, vinylmercuric xanthate decomposes after a few days accompanied by the deposition of a grey-black precipitate. Thermal decomposition at 115 to $125{ }^{\circ} \mathrm{C}$ under a reduced pressure of $25 \mathrm{~mm}$ gave

1 G. SPengler and A. Weber, Brennstoff-Chem. 40, 56 [1959].

2 R. Felumb, Bull. Soc. chim. France, 1957, 890.

3 L. J. Bellamy, "The Infrared Spectra of Complex Molecules", 2nd. Ed., Methuen, London 1956, p. 356 and p. 116. 4 See reference 3., p. 347.

5 E. Lieber, C. N. R. Rao and J. Ramachandran, Spectrochim. Acta [London] 13, 296 [1959]. a yellow oil $\left(n_{\mathrm{D}}^{20} 1.5705\right)$, which is believed to be vinylxanthate, $\mathrm{C}_{2} \mathrm{H}_{5} \mathrm{OCSSCH}=\mathrm{CH}_{2}$. The infrared spectrum of the compound is characterized by two sharp absorption bands at $8.22 \mu(\mathrm{C}=\mathrm{S})^{2,3}$ and $9.46 \mu$ $(\mathrm{C}-\mathrm{O}-\mathrm{C})^{3}$.

Vinylmercuric acetate also reacts with sodium thiocyanate to yield vinylmercuric thiocyanate, m. p. 138.5 to $139^{\circ}$. The infrared spectrum exhibits a strong $\mathrm{CH}_{2}=\mathrm{CHHgO}_{2} \mathrm{CCH}_{3}+\mathrm{NaSCN} \stackrel{\mathrm{EtOH}}{\longrightarrow} \mathrm{CH}_{2}=\mathrm{CHH}_{\mathrm{gSCN}}$ absorption band at $4.83 \mu$, due to the $\mathrm{S}-\mathrm{C} \equiv \mathrm{N}$ vibration $^{4}$. (Found: C, 12.39; H, 1.08; N, 5.13; S, 11.38. $\mathrm{C}_{3} \mathrm{H}_{3} \mathrm{HgNS}$ requires $\mathrm{C}, 12.61 ; \mathrm{H}, 1.05 ; \mathrm{N}, 4.90 ; \mathrm{S}$, 11.20.)

Thermal decomposition at $100-120^{\circ}$ under a reduced pressure of $100 \mathrm{~mm}$. gave a colorless liquid of repulsive odor, $n_{\mathrm{D}}^{20}$ 1.5042. The infrared spectrum of this material showed strong absorptions at 4.58 $(\mathrm{N}=\mathrm{C}=\mathrm{S}), 4.63(\mathrm{~S}-\mathrm{C} \equiv \mathrm{N})$ and $4.82 \mu(\mathrm{N}=\mathrm{C}=\mathrm{S})^{5}$, indicating the presence of vinylisothiocyanate (lit. ${ }^{6}$, b. p. $46^{\circ}$ at $100 \mathrm{~mm}$., $\left.n_{\mathrm{D}}^{33} 1.505\right)$ and vinylthiocyanate (lit. ${ }^{7}$, b. p. $54^{\circ}$ at $62 \mathrm{~mm} ., n_{\mathrm{D}}^{20}$ 1.4880). According to a vapor phase chromatographic analysis, the mixture consisted of 30 per cent $\mathrm{CH}_{2}=\mathrm{CHN}=\mathrm{C}=\mathrm{S}$ and 66 per ecnt $\mathrm{CH}_{2}=\mathrm{CHSC} \equiv \mathrm{N}$.

As already described in the previous communication $^{8}$, the reaction of divinylmercury with $N$-bromoimides results in the formation of $N$-vinylmercuric imides. The latter can also be obtained from vinylmercuric salts and imides in the presence of base ${ }^{9}$. Thus, treatment of an alcoholic solution of phthalimide with a solution of vinylmercuric halide in alco-

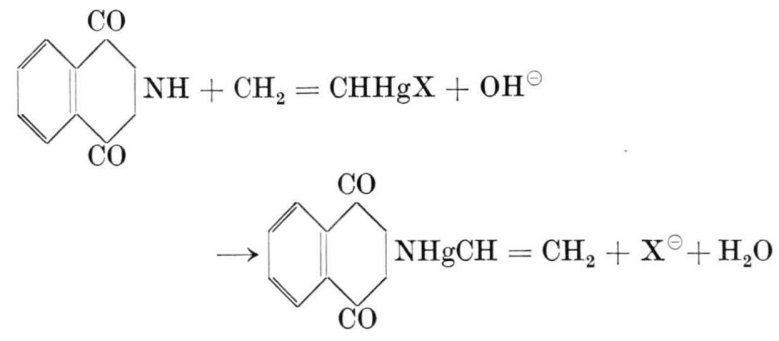

6 U.S. Patent 2,757,190, July 31, 1956.

7 J. C. H. Hwa, J. Amer. chem. Soc. 81, 3604 [1959].

8 E. Tobler and D. J. Foster, Z. Naturforschg. 17 b, 135 [1962].

9 M. S. Whelen, "Metal-Organic Compounds", Advances in Chemistry, Series 23, ACS, Washington 1959, p. 84. 
holic potassium hydroxide yielded $N$-vinylmercuric phthalimide, m. p. $134.5-135^{\circ}$ (from $\mathrm{CCl}_{4}$ ). The compound was found to be identical with the one obtained previously ${ }^{8}$, as indicated by a mixed m. p. and a comparison of the infrared spectra.

Numerous organomercury compounds have been prepared by passing acetylene into solutions of various mercury salts ${ }^{10}$. Bubbling acetylene through alkaline solutions of vinyl- or cyclopropylmercuric halides results in the precipitation of bis (vinylmercuric) acetylene $\left(\mathrm{R}=\mathrm{CH}_{2}: \mathrm{CH}-\right)$. (Found: $\mathrm{C}, 15.3 ; \mathrm{H}, 1.21$;

10 G. E. Coates, "Organometallic Compounds", Methuen, London 1956, p. 50.

$$
\begin{aligned}
2 \mathrm{RHgX}+\mathrm{CH} \equiv \mathrm{CH}+2 \mathrm{OH} \ominus \\
\longrightarrow \mathrm{RHgC} \equiv \mathrm{CHgR}+2 \mathrm{X} \ominus \boldsymbol{z}+\mathrm{H}_{2} \mathrm{O}
\end{aligned}
$$

$\mathrm{Hg}$, 83.5. $\mathrm{C}_{6} \mathrm{H}_{6} \mathrm{Hg}$ requires $\mathrm{C}, 15.1 ; \mathrm{H}, 1.25 ; \mathrm{Hg}$, 83.7) and bis (cyclopropylmercuric) acetylene ( $\mathrm{R}=$ cyclopropyl). (Found: C, 18.82; H, 2.08; Hg, 78.9. $\mathrm{C}_{8} \mathrm{H}_{10} \mathrm{Hg}$ requires $\mathrm{C}, 18.95 ; \mathrm{H}, 1.99 ; \mathrm{Hg}, 79.1$ ) respectively. Both compounds are white, crystalline solids, which do not melt below $300^{\circ}$ and are quite insoluble in organic solvents. Hydrochloric acid cleavage of these organomercury acetylenes gave mercuric chloride, acetylene and the corresponding hydrocarbon (ethylene and cyclopropane, respectively).

$\mathrm{RHgC} \equiv \mathrm{CHgR}+4 \mathrm{HCl} \longrightarrow \mathrm{CH} \equiv \mathrm{CH}+2 \mathrm{RH}+2 \mathrm{HgCl}_{2}$.

\section{Rückstoßfreie Gammastrahl-Resonanzabsorption an den ${ }^{57} \mathrm{Fe}$-Atomen des Haemins und Haemoglobins}

\section{Von W. Karger}

Physiologisches Institut der Universität Göttingen

(Z. Naturforschg. 17 b, 137-139 [1962] ; eingegangen am 19. August 1961)

Mössbauer ${ }^{1}$ entdeckte 1958 einen für die Messung kleinster Energiedifferenzen wichtigen Effekt: Angeregte Atomkerne, die durch $\gamma$-Strahlung von der ersten Anregungsstufe in den quantenmechanischen Grundzustand übergehen, können unter geeigneten Bedingungen den ausgesandten Quanten die volle Energie des Übergangs mitgeben. Im allgemeinen wird dies aus Gründen der Impulserhaltung nicht eintreten, - der emittierende Kern erfährt einen Rückstoß. Die hierfür aufgewandte Energie fehlt dem Quant; seine Frequenz ist entsprechend vermindert. Eine analoge Überlegung gilt für den Akt der Absorption. MössbaUer zeigte nun, daß der Rückstoß am einzelnen Kern vermieden werden kann, wenn Emitter- und Absorberatome jeweils in genügend fester Bindung in einem Kristallgitter vorliegen, da dann der Kristallverband als Ganzes den Impuls aufnehmen kann. Somit war die Möglichkeit der Beobachtung einer Resonanzabsorption mit der vollen Schärfe der natürlichen Linienbreite gegeben. Speziell am Kern des Eisenisotops ${ }^{57} \mathrm{Fe}$ ist dies gut durchführbar, wie von Pound und Rebka ${ }^{2}$ und von Schiffer und Marshall $^{3}$ gezeigt wurde. Die geringe Über-

1 R. L. Mössbauer, Z. Physik 151, 124 [1958] ; Naturwissenschaften 45, 538 [1958]; Z. Naturforschg. 14 a, 211 [1959].

2 R. V. Pound u. G. A. Rebka, Physic. Rev. Lett. 3, 554 u. 440 [1959].

3 J. P. Schiffer u. W. Marshall, Physic. Rev. Lett. 3, 556 [1959].

4 S. L. Ruby, L. M. Epstein u. K. H. Sun, Rev. sci. 31, 580 [1960].

5 K. G. Wertheim, J. appl. Physics, Suppl. 32, 110 [1961].

${ }^{6}$ I. Solomon, C. R. hebd. Séances Acad. Sci. 250, 3828 [1960]; 251, 2675 [1960].

7 G. DePasquali, H. Frauenfelder, S. Margulies u. R. N. Peacock, Phys. Rev. 4, 71 [1960]. gangsenergie des 1 . angeregten Zustands dieses Kerns, die $1,44 \cdot 10^{4}[\mathrm{eV}]$ beträgt, hat auch eine kleine Rückstoßenergie von $2 \cdot 10^{-3}[\mathrm{eV}]$ zur Folge. Im metallischen Eisen wirkt sich die hohe D e b y e - Temperatur des Kristallgitters vorteilhaft in dem Sinne aus, daß auf eine Abkühlung auf tiefe Temperaturen verzichtet werden kann, da ein erheblicher Prozentsatz der ${ }^{57} \mathrm{Fe}$ Kerne noch bei Raumtemperatur zur rückstoßfreien Emission oder Absorption befähigt ist. Auch in Eisenverbindungen wie $\mathrm{Fe}_{2} \mathrm{O}_{3}, \mathrm{FeS}_{2}, \mathrm{FeSO}_{4}, \mathrm{~K}_{4} \mathrm{FeCN}_{6}$ wurde bei normalen Temperaturen noch ein merklicher M ö s s b a u e r - Effekt beobachtet ${ }^{4,5,6}$. Hinsichtlich der ferner aufgefundenen Besonderheiten (Linienaufsplitterung durch $\mathrm{Z}$ e e man-Effekt, Verschiebung der Linienlage entsprechend der Art und Stärke der chemischen Bindung) muß auf die Originalarbeiten und umfassendere Darstellungen ${ }^{7-13}$ verwiesen werden.

Nun ist die biologisch wichtigste Eisenverbindung das Hämoglobin. Es erschien daher einer eingehenden Überlegung und Prüfung wert, ob die Möglichkeit einer Resonanzabsorption für die zentralen Eisenatome inmitten der vier prosthetischen Gruppen des Hämoglobins, den Hämen, gegeben sein könnte und ein vorhandener Effekt innerhalb der Grenzen der Beobachtungsmöglichkeiten liegen würde. Denn gegenüber den anderen untersuchten Verbindungen war zunächst als entschiedener Nachteil die außerordentlich geringe Eisenkonzentration zu bewerten. Von den $4 \mathrm{Fe}$-Atomen im Molekül (Mol.-Gew. des Hämoglobins = 68000 ) können, entsprechend der natürlichen Isotopenhäufigkeit, nur $2,2 \%{ }^{57} \mathrm{Fe}$-Atome sein, von denen wiederum

8 G. J. Perlow, S. S. Hanna, M. Hamermesh, C. Littlejohn, D. H. Vincent, R. S. Preston u. J. Heberle, Physic. Rev. Lett. 4, 74 [1959].

9 W. M. Visscher, Ann. Physics 9, 194 [1960].

10 H. J. Lipkin, Ann. Physics 9, 332 [1960].

11 E. Cotron, J. Physique, Radium 21, 265 [1960].

12 A. Abragam, Vorlesungsmanuskript: L'Effet Mös s $\mathrm{b}$ a u e $\mathrm{r}$ et ses applications à l'étude des champs internes, Paris 1960.

13 I. Y. Krause u. G. Lüders, Naturwissenschaften 47, 532 [1960]. 